\title{
Don't Optimize Existing Protocols, Design Optimizable Protocols
}

\author{
Jiayue He, Jennifer Rexford and Mung Chiang \\ Princeton University, Princeton, New Jersey, United States \\ jhe@princeton.edu, jrex@princeton.edu, chiangm@princeton.edu
}

This article is an editorial note submitted to CCR. It has NOT been peer reviewed. Authors take full responsibility for this article's technical content. Comments can be posted through CCR Online.

\begin{abstract}
As networks grow in size and complexity, network management has become an increasingly challenging task. Many protocols have tunable parameters, and optimization is the process of setting these parameters to optimize an objective. In recent years, optimization techniques have been widely applied to network management problems, albeit with mixed success. Realizing that optimization problems in network management are induced by assumptions adopted in protocol design, we argue that instead of optimizing existing protocols, protocols should be designed with optimization in mind from the beginning. Using examples from our past research on traffic management, we present principles that guide how changes to existing protocols and architectures can lead to optimizable protocols. We also discuss the trade-offs between making network optimization easier and the overhead these changes impose.
\end{abstract}

Categories and Subject Descriptors

C.2.1 [Computer Communication Networks]:

Network Architecture and Design

General Terms

Design, Management, Performance

\section{INTRODUCTION}

Network management is the continuous process of monitoring a network to detect and diagnose problems, and of configuring protocols and mechanisms to fix problems and optimize performance. Traditionally, network management has been largely impenetrable to the research community since many of the problems appear both complex and ill-defined. In the past few years, the research community has made tremendous progress casting many important network management problems as optimization problems. Network optimization involves satisfying network management objectives by setting the tunable parameters that control network behavior. Solving an optimization problem involves optimizing an objective function subject to a set of constraints. It is well-established that convexity is a watershed between hard and easy optimization problems. Unfortunately, many optimization problems that arise in data networks are nonconvex. Consequently, they are computationally intractable, with many local optima that are suboptimal.

In this paper, we argue that the difficulty of solving the key optimization problems is an indication that we may need to revise the underlying protocols, or even the architectures, that lead to these problem formulations in the first place. We advocate the design of optimizable networks - network architectures and protocols that lead to easy-to-solve optimization problems and consequently, optimal solutions. Indeed, the key difference between "network optimization" and "optimizable networks" is that the former refers to solving a given problem (induced by the existing protocols and architectures) while the latter involves formulating the "right" problem (by changing protocols or architectures accordingly).

The changes to protocols and architectures can range from minor extensions to clean-slate designs. In general, the more freedom we have to make changes, the easier it would be to create an optimizable network. On the other hand, the resulting improvements in network management also need to balanced against other considerations such as scalability and extensibility, and must be made judiciously. To make design decisions, it is essential to quantify the trade-off between making network-management problems easier by changing the problem statement and the extra overhead the resulting protocol imposes on the network.

Network optimization has had a particularly large impact in the area of traffic management, which controls the flow of traffic through the network. Today, this spans across congestion control, routing and traffic engineering. In Section 2, we describe how optimization is used in traffic management today. In Section 3, we illustrate design principles which we have uncovered through our own research experiences on traffic management. Traffic management is an extremely active area of research, but we will not address related work in this paper since these examples are included to serve as illustrations of general principles. In Section 4, we discuss other aspects of traffic management, such as interdomain routing and active queue management, where the problems are even more challenging. We also examine the trade-off between performance achieved and overhead imposed when designing optimizable protocols. We conclude and point to future work in Section 5 .

\section{TRAFFIC MANAGEMENT TODAY}

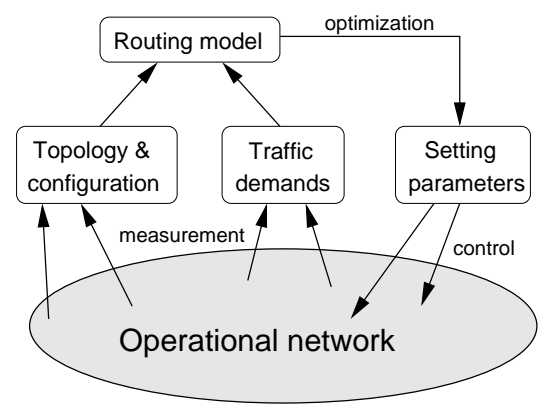

Figure 1: Components of the route optimization framework.

In this section, we introduce how optimization is used in the context of traffic management inside a single Autonomous System 
(AS). Traffic management has three players: users, routers, and operators. In today's Internet, users run TCP congestion control to adapt their sending rates at the edge of the network based on packet loss. Congestion control has been reverse engineered to be implicitly solving an optimization problem, $[1,2,3]$. Inside the network, operators tune parameters in the existing routing protocols to achieve some network-wide objective in a process called traffic engineering, see Figure 1.

\subsection{Traffic Engineering}

Inside a single AS, each router is configured with an integer weight on each of its outgoing links, as shown in Figure 2. The routers flood the link weights throughout the network and compute shortest paths as the sum of the weights. Each router uses this information to construct a table that drives the forwarding of each IP packet to the next hop in its path to the destination. These protocols view the network inside an AS as a graph $G(R, L)$ where each router is a node $r \in R$ and each directed edge is a link $l \in L$ between two routers. Each unidirectional link has a fixed capacity $c_{l}$, as well as a configurable weight $w_{l}$. The outcome of the shortestpath computation can be represented as the proportion $P_{i, j, l}$ of the traffic from router $i$ to router $j$ that traverses the link $l$.

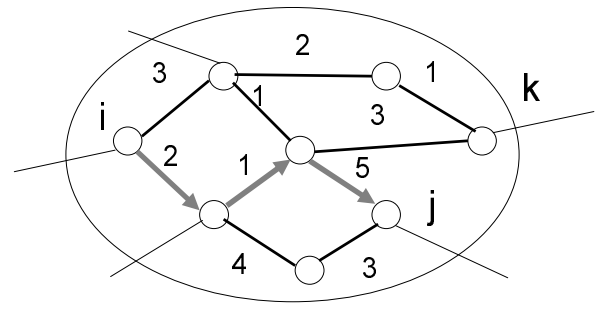

Figure 2: Network topology with link weights for shortest path routing.

Operators set the link weights in intradomain routing protocols in a process called traffic engineering. The selection of the link weights $w_{l}$ should depend on the offered traffic, as captured by a matrix $M_{i, j}$ that represents the rate of traffic entering at router $i$ that is destined to router $j$. The traffic matrix can be computed based on traffic measurements or may represent explicit subscriptions or reservations from users. Given the traffic matrix $M_{i, j}$ and link weights $w_{l}$, the volume of traffic on each link $l$ is $y_{l}=$ $\sum_{i, j} M_{i, j} P_{i, j, l}$, the proportion of traffic that traverses link $l$ summed over all pairs of routers. An objective function can quantify the "goodness" of a particular setting of the link weights. For traffic engineering, the optimization considers a network-wide objective of minimizing $\sum_{l} f\left(y_{l} / c_{l}\right)$ for a convex function $f$ that penalizes solutions that have heavily-loaded links.

So far, we have covered the impact of link weights inside an AS. When a network, such as an Internet Service Provider (ISP) backbone, can reach a destination through multiple egress points, a routing change inside the AS may change how traffic leaves the AS. Each router $i$ typically selects the closest egress point $e$ out of a set $E_{d}$ of egress points which can reach destination $d$, in terms of the intradomain link weights $w_{l}$, in a practice known as early-exit or hot-potato routing [4]. In the example in Figure 2, suppose a destination $d$ is reachable via egress points $j$ and $k$. Then traffic from $i$ exits via $k$ rather than $j$ since the intradomain path cost from $i$ to $k$ is smaller. If the traffic from $i$ encounters congestion along the downstream path from $k$ in Figure 2, the network operators could tune the link weights to make the path through $j$ appear more attractive. Controlling where packets leave the network, and preventing large shifts from one egress point to another, is an important part of engineering the flow of traffic in the network. Models can capture the effects of changing the link weights on the intradomain paths and the egress points, but identifying good settings of the weights is very difficult.

\subsection{Pros and Cons of Traffic Management}

Traffic management today has several strengths. First, routing depends on a very small amount of state per link i.e., link weights. In addition, forwarding is done hop-by-hop, so that each router decides independently how to forward traffic on its outgoing links. Second, routers only disseminate information when link weights or topology change. In addition, TCP congestion control is based only on implicit feedback of packet loss loss and delay, rather than explicit messages from the network. Third, the selection of link weights can depend on a wide variety of performance and reliability constraints. Fourth, hot-potato routing reduces internal resource usage (by using the closest egress point), adapts automatically to changes in link weights, and allows routers in the AS to do hopby-hop forwarding toward the egress point. Last but not least, the decoupling of congestion control and traffic engineering reduces complexity through separation of concerns.

On the other hand, today's protocols also have a few shortcomings. To start with, optimizing the link weights in shortest-path routing protocols based on the traffic matrix is NP-hard, even for simplest of objective functions [5]. In practice, local-search techniques are are used for selecting link weights [5]; however, the computation time is long and, while the solutions are frequently good [5], the deviation from the optimal solution can be large. Finding link weights which work well for egress point selection is even more challenging, as this adds even more constraints on how the weights are set.

There are other limitations to today's traffic management. The network operator can only indirectly influence how the routers forward traffic, through the setting of the link weights. Further, traffic engineering is performed assuming that the offered traffic is inelastic. In reality, end hosts adapt their sending rates to network congestion, and network operators adapt the routing based on measurements of the traffic matrix. Although congestion control and routing operate independently, their decisions are coupled. The joint system is stable, but often suboptimal [6]. Furthermore, traffic engineering does not necessarily adapt on a small enough timescale to respond to shifts in user demands. In addition to timescale alternatives, there are also choices as to geographically which part of traffic management work should be carried out inside the network, and which by the sources. These limitations suggest that revisiting architectural decisions is a worthy research direction.

\section{DESIGN OPTIMIZABLE PROTOCOLS}

In this section, we illustrate three design principles through proposed protocols. The three principles also correspond to the three parts of an optimization problem formulation: objective, variables and constraints. In a generic optimization problem formulation, the objective is to minimize $f(x)$ over the variable $x$, subject to constraints on $x$ :

$$
\begin{array}{ll}
\text { minimize } & f(x) \\
\text { subject to } & x \in S \\
\text { variable } & x
\end{array}
$$

From optimization theory, it is well established that a local optimum of (1) is also a global optimum, which can be found in polynomial time and often very fast, if $S$ is a convex set and $f$ is a convex 
function ${ }^{1}$. In other words, a convex optimization problem leads to both tractability and optimality. Due to single-path routing, an artifact of the current system, the constraint set is not convex for most traffic management problems. In our first example, we tackle this problem head-on by changing the shape of the constraint set. In our second example, we avoid the problem because the particular problem formulation falls under a special class of integer programming problems. In our third example, we change the system to allow routing to be per path multi-commodity flow, so that decomposition techniques can be applied to derive stable and fast-timescale interaction between routing and congestion control.

\subsection{Changing the Shape of the Constraint Set}

Some optimization problems involve integer constraints, which are not convex, making them intractable and their solutions suboptimal. Relaxing the integer constraint to approximate a convex constraint can lead to a more tractable problem and a smaller optimality gap. This is the case in link-weight setting problem where the network usually has a single shortest path from $i$ to $j$, resulting in $P_{i, j, l}=1$ for all links $l$ along the path, and $P_{i, j, l}=0$ for the remaining links. An OSPF or IS-IS router typically splits traffic evenly along one or more outgoing links along shortest paths to the destination, allowing for limited fractional values of $P_{i, j, l}$, but the constraint set is still highly nonconvex. The ability to split traffic arbitrarily over multiple paths would make the constraints convex, i.e., $P_{i, j, l} \in[0,1]$. The downside is this approach would sacrifice the simplicity of OSPF and IS-IS, where routers compute paths in a distributed fashion based on a small amount of configuration state.

Rather than supporting arbitrary splitting, a recent proposal advocates small extensions to OSPF and IS-IS to split traffic over multiple paths [7]. Under this proposal, the routers forward traffic on multiple paths, with exponentially diminishing proportions of the traffic directed to the longer paths. For example, in Figure 2 , there are two outgoing links from $i$, with link weights $(2,3)$, the load on each path would be proportional to $\left(e^{-2}, e^{-3}\right)$. Under this formulation, both link weights and the flow splitting ratios are variables. This enlarges the constraint set, and the resulting constraints are much easier to approximate with convex constraints. Consequently, the link-weight tuning problem is tractable, i.e., can be solved much faster than the local search heuristics today. In addition, the modified protocol is closer to optimal, i.e., makes more efficient use of link capacities, and is more robust to small changes in the path costs. By changing the constraint set, the work in [7] retains the simplicity of link-state routing protocols and hop-by-hop forwarding, while inducing an optimization problem that is both faster to solve and lead to smaller optimality gap.

\subsection{Adding Variables to Decouple Constraints}

Some optimization problems can involve many tightly-coupled constraints, making it difficult to find a feasible solution. Introducing extra variables can decouple the constraints, and increase the size of the feasible region. As an example, setting the link weights is highly constrained, since the weights are used to compute both the forwarding paths between the routers inside the domain and the egress points where the traffic leaves the domain. Weakening the coupling between intradomain routing and egress-point selection is the key to simplifying the optimization problem and improving network performance.

Rather than selecting egress points $e$ from ingress router $i$ based only on the intradomain path costs $w_{i, e}$ (sum of all link weights $w_{l}$

\footnotetext{
${ }^{1}$ A convex set $S$ is defined as if $x, y \in S$, then $\theta x+(1-\theta) y \in S$, for all $\theta \in[0,1]$. A function $f$ is a convex function if domain of $f$ is a convex set and $f(\theta x+(1-\theta) y) \leq \theta f(x)+(1-\theta) f(y)$.
}

on the path from $i$ to $e$ ), a variable $q_{i, p, e}$ is introduced for router $i$, across all prefixes $p$ and egress points $e$. To support flexible policy while adapting automatically to network changes, the metric $q_{i, p, e}$ includes both configurable parameters and values computed directly from a real-time view of the topology. In particular, $q_{i, p, e}=\alpha_{i, p, e} w_{i, e}+\beta_{i, p, e}$ where $\alpha$ and $\beta$ are configurable values [8]. The first component of the equation supports automatic adaptation to topology changes, whereas the second represents a static ranking of egress points per ingress router. Providing separate parameters for each destination prefix allows even greater flexibility, such as allowing delay-sensitive traffic to use the closest egress point while preventing unintentional shifts in the egress points for other traffic.

In general, the resulting integer multicommodity-flow problem is still nonconvex and consequently intractable. This problem formulation happen to correspond to a very special subset of integer programming problems where relaxing the integrality constraints $x(i, e, p) \in 0,1$ to simply $x(i, e, p) \geq 0$ would still produce integer solutions [9], thus side-stepping the convexity issue. Overall, by increasing the degrees of freedom, a management system can set the new parameters under a variety of constraints that reflect the operators' goals for the network [8]. Not only does the network become easier to optimize, but the performance improves as well, due to the extra flexibility in controlling where the traffic flows.

\subsection{Combining Objectives to Derive Protocols}

In a system, there can be multiple interacting optimization problems with different objectives. Combining the objectives of multiple problems can allow for a better solution to the overall problem. In today's traffic management system, congestion control and traffic engineering have different objectives. Congestion control tries to maximize aggregate user utility, and as a result tends to push traffic into the network so that multiple links are used at capacity. In contrast, traffic engineering uses a link cost function which heavily penalizes solutions with bottleneck links. One way to combine the objectives of traffic engineering and congestion control is to construct a weighted sum of utility and link cost functions as the overall objective for traffic management [6].

In [6], we revisit the division of labor between users, operators and routers. In this case, we allow for a per path multi-commodity flow solution, hence resulting in a convex problem, and opens up many standard optimization techniques such as dual decomposition that derive distributed and iterative solutions. Decomposition is the process of breaking up a single optimization problem into multiple ones that can be solved independently. Decomposing the overall traffic management optimization problem, a distributed protocol is derived that splits traffic over multiple paths, where the splitting proportions depend on feedback from the links. The solution is called DATE (Distributed Adaptive Traffic Engineering). By embedding the management objectives in the protocols, the link-cost function is now automated incorporated by the links themselves as part of computing the feedback sent to the edge routers, rather than by the network-management system. As such, the networkmanagement system merely specifies the link cost function, and does not need to adapt the configuration of the routers over time.

\section{OPEN CHALLENGES IN TRAFFIC MANAGEMENT OPTIMIZATION}

The principles introduced in the previous section are a useful first step towards designing optimizable protocols, but are by no means comprehensive. The merits of proposed optimizable protocols should always be balanced with any extra overhead in practical 
implementation and robustness to changing network dynamics. In addition, the principles introduced in the previous section focuses on intradomain traffic management, and do not address all the challenges in end-to-end traffic management. Finally, when deriving new architectures, the balance between performance and other factors is even more delicate.

\subsection{Performance vs. Overhead Trade-off}

Characterizing a network architecture in terms of the tractability of network-management problems is just one piece of a complex design puzzle. The design of optimizable networks introduces tension between the ease of network optimizability and the overhead on network resources. Some of the architectural decisions today make the resulting protocols simple. For example, protocols which rely on implicit feedback e.g., TCP congestion control, do not have message passing overhead. Further, hop-by-hop forwarding does not depend on the upstream path, requiring less processing at the individual routers. It would be desirable to capture such notions of simplicity mathematically, so we can learn to derive optimizable protocols which retain them.

Our example in Section 3.1 manages to retain the simplicity of hop-by-hop forwarding while resulting in a tractable optimization problem. In this particular case, optimality gap was significantly reduced with very little extra overhead. However, some approaches make the protocol more optimizable at the expense of additional overhead. For example, adding flexibility in egress-point selection in Section 3.2 introduces more parameters that the networkmanagement system must set. Similarly, revisiting the division of functionalities in Section 3.3 leads to a solution that requires explicit feedback from the links. Imposing extra overhead on the network may be acceptable, if the improvement in performance is sufficiently large.

Furthermore, ensuring a completely tractable optimization problem is sometimes unnecessary. An NP-hard problem may be acceptable, if good heuristics are available. For striking the right trade-offs in the design of optimizable networks, it is important to find effective ways to quantify the acceptable amount of deviation from the optimal solution. There are also well-established, quantitative measures of the notions of how easily-solvable an optimization is. These quantitative measures can help determine how much the protocols and architectures need to change to better support network management.

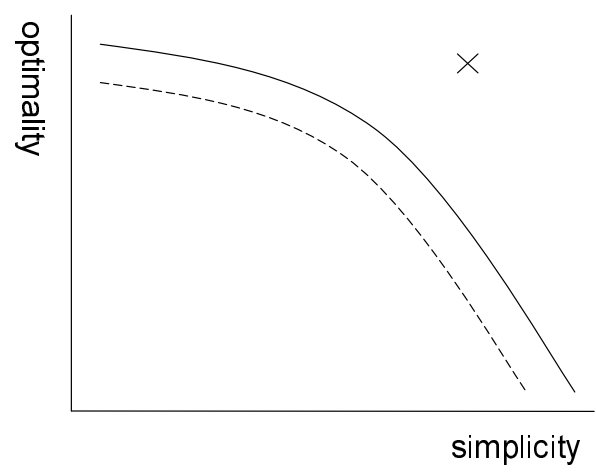

Figure 3: Different trade-off curves in the optimality versus simplicity in design space. The dotted line is the original tradeoff curve, the solid line and the cross are possible when underlying assumptions are perturbed.

The protocols today are designed with certain assumptions in mind, e.g., single-path routing and hop-by-hop forwarding. Some of these assumptions cause the resulting optimization problem to be intractable e.g., single-path routing, while others do not, e.g., hop-by-hop forwarding. By perturbing the underlying assumptions in today's protocol, we can achieve a different curve or a different point in the trade-off space of optimality versus simplicity, see Figure 3. This curve or point could be strictly better than today's protocols, or at least offer different properties. Therefore, it's worth exploring the alternatives, even if at the end the decision is to keep the original protocol and architectures. In order to choose between protocol designs, the key is to gain a deeper understanding of the trade-offs. As such, we believe that design for optimizability can be a promising, new interdisciplinary area between the systems and theory communities.

\subsection{End-to-End Traffic Management}

Our examples thus far focused on optimization problems in intradomain traffic management. Routing within a single domain side-steps several important issues that arise in other aspects of data networking, for several reasons:

- A single domain has the authority to collect measurement data (such as the traffic and performance statistics) and tune the protocol configuration (such as the link weights).

- The routing configuration changes on the timescale of hours or days, allowing ample time to apply more computationally intensive solution techniques.

- The optimization problems consider highly aggregated information, such as link-level performance statistics or offered load between pairs of routers.

When these assumptions do not hold, the resulting optimization problems become even more complicated, as illustrated by the following two examples.

Optimization in interdomain traffic management: In the Internet, there are often multiple Autonomous Systems (AS) in the path between the sender and the receiver. Each AS does not have full view of the topology, only the paths which are made visible to it through the routing-protocol messages exchanged in the Border Gateway Protocol (BGP). In addition, each AS has a set of private policies that reflect its business relationships with other ASes. Without full visibility and control, it is difficult to perform interdomain traffic management. For example, to implement DATE in the Internet, the ASes would need to agree to provide explicit feedback from the links to the end hosts or edge routers, and trust that the feedback is an honest reflection of network conditions. Extending BGPs to allow for multiple paths would simplify the underlying optimization problem, but identifying the right incentives for ASes to deploy a multipath extension to BGP remains an open question.

Optimization in active queue management: A router may apply active queue management schemes like Random Early Detection [10] to provide TCP senders with early feedback about impending congestion. RED has many configurable parameters to be selected by network operators, e.g.,, queue-length thresholds and maximum drop probability. Unfortunately, predictive models for how the tunable parameters affect RED's behavior remain elusive. In addition, the appropriate parameter values may depend on a number of factors, including the number of active data transfers and the distribution of round-trip times, which are difficult to measure on high-speed links. Recent analytic work demonstrates that setting RED parameters to stabilize TCP is fundamentally difficult [11]. It is appealing to explore alternative active-queue management schemes that are easier to optimize, including self-tuning 
algorithms that do not require the network-management system to adjust any parameters.

From these two examples, it is clear that there remains open challenges in end-to-end traffic management. Outside the context of traffic management, network optimization's role is even less understood. We argue for a principled approach in tackling these challenges, so that in time, protocol design can more less of an art and more of a science.

\subsection{Placement of Functionality}

The challenges are not just limited to protocols, but extends to architectural decisions regarding the placement of functionality. Architecturally, the DATE example represents one extreme where most of computation and coordination is moved into the distributed protocols that run in the routers. In the context of Figure 1, this means much of the measurement, control and optimization is pushed down into the network. One can consider another extreme, where the network-management systems bear all the responsibility for adapting to changes in network conditions, as in [12]. Both approaches redefine the division of labor between the management system and the routers, where one moves most of the control into the distributed protocols and the other has the management systems directly specify how the routers handle packets.

In some cases, having the management system bear more responsibility would be a natural choice. For example, if an optimization problem is fundamentally difficult, consequently leading to distributed solutions that are complicated or suboptimal, or both. Unlike the routers, a management system has the luxury of a global view of network conditions and the ability to run centralized algorithms for computing the protocol parameters. Today's traffic engineering uses the centralized approach and allows operators to tailor the objectives to the administrative goals of the network. This leads to a more evolvable system, where the objective function and constraints can differ from one network to another, and change over time. In addition, the operators can capitalize on new advances in techniques for solving the optimization problems, providing an immediate outlet for promising research results.

The network-management system can apply centralized algorithms based on a global view of network conditions, at the expense of a slower response based on coarse-grain measurements. Yet some parts of traffic management, such as detecting link failures and traffic shifts, must occur in real time. In order to understand which functions must reside in the routers to enable adaptation on a sufficiently small time-scale, it is important to quantify the loss in performance due to slower adaptation. For functions which require fast adaptation, an architecture where end user load balance across multiple paths would be desirable. For functions that can operate on a slower timescale, the control of flow distribution can be left to operators. In general, determining the appropriate division of labor between the network elements and the management systems is an avenue for future research.

\section{CONCLUSIONS AND FUTURE WORK}

In recent years, optimization has played an increasingly important role in network management. In this paper, we argue that, instead of just trying to optimize existing protocols, protocols should be designed for the ease of optimization. If a set of architectures and protocols lead to intractable optimization problems for network management, we argue that, instead of trying to solve these problems by ad hoc heuristics, we should revisit some of the underlying assumptions in the architectures and protocols. Such explorations can lead to easier network optimization problems and may provide superior simplicity-optimality tradeoff curves.
Drawing from our own research experiences in traffic management, we propose three guiding principles for making optimizable protocols which correspond to three aspects of an optimization problem i.e., constraints, variables and objective. First, changing the constraint set can turn an NP-hard optimization problem into an easy problem and reduce the optimality gap. Second, increasing degrees of freedom (by introducing extra parameters) can break tightly coupled constraints. Finally, embedding management objectives in the protocol can lead to alternative architectures. Still, protocols changes must be made judiciously to balance the gain in performance with the extra consumption of network resources.

Ultimately, the design of manageable networks raises important architectural questions about the appropriate division of functionalities between network elements and the systems that manage them. This paper represents a first step toward identifying design principles that can guide these architectural decisions. The open challenges which remain suggest that the design of manageable networks may continue to be somewhat of an art, but hopefully one that will be guided by more and more design principles. We believe that providing a new, comprehensive foundation for the design of manageable networks is an exciting avenue for future research.

\section{Acknowledgment}

We would like to thank Constantine Dovrolis, Nick Feamster, Renata Teixeira and Dahai Xu for their feedback on earlier drafts. This work has been supported in part by NSF grants CNS-0519880 and CCF-0448012, and DAPRA Seedling W911NF-07-1-0057.

\section{REFERENCES}

[1] F. P. Kelly, A. Maulloo, and D. Tan, "Rate control for communication networks: Shadow prices, proportional fairness and stability," $J$. of Operational Research Society, vol. 49, pp. 237-252, March 1998.

[2] S. H. Low, "A duality model of TCP and queue management algorithms," IEEE/ACM Trans. Networking, vol. 11, pp. 525-536, August 2003.

[3] R. Srikant, The Mathematics of Internet Congestion Control. Birkhauser, 2004.

[4] R. Teixeira, A. Shaikh, T. Griffin, and J. Rexford, "Dynamics of hot-potato routing in IP networks," in Proc. ACM SIGMETRICS, June 2004.

[5] B. Fortz and M. Thorup, "Optimizing OSPF weights in a changing world," IEEE J. on Selected Areas in Communications, vol. 20, pp. 756-767, May 2002.

[6] J. He, M. Bresler, M. Chiang, and J. Rexford, "Towards multi-layer traffic engineering: Optimization of congestion control and routing," IEEE J. on Selected Areas in Communications, June 2007.

[7] D. Xu, M. Chiang, and J. Rexford, "DEFT: Distributed exponentially-weighted flow splitting," in Proc. IEEE INFOCOM, May 2007.

[8] R. Teixeira, T. Griffin, M. Resende, and J. Rexford, "TIE Breaking: Tunable Interdomain Egress Selection," in Proc. CoNEXT, October 2005.

[9] A. Ozdaglar and D. P. Bertsekas, "Optimal Solution of Integer Multicommodity Flow Problems with Application in Optical Networks,"

[10] S. Floyd and V. Jacobson, "Random early detection gateways for congestion avoidance," IEEE/ACM Trans. Networking, vol. 1, pp. 397-413, August 1993.

[11] S. H. Low, F. Paganini, J. Wang, and J. C. Doyle, "Linear stability of TCP/RED and a scalable control," Computer Networks, vol. 43, pp. 633-647, December 2003.

[12] A. Greenberg, G. Hjalmtysson, D. A. Maltz, A. Meyers, J. Rexford, G. Xie, H. Yan, J. Zhan, and H. Zhang, "A clean slate 4D approach to network control and management," ACM SIGCOMM Computer Communication Review, October 2005. 